\title{
Network pharmacology study on the mechanism of Qiangzhifang in the treatment of panic disorder
}

\author{
Run Zhao ${ }^{1 \#}$, Pulin Liu ${ }^{1}$, Anran Song ${ }^{1}$, Jianmin Liu ${ }^{2}$, Qian Chu ${ }^{1}$, Yingnan Liu ${ }^{1}$, Yunyun Jiang ${ }^{1}$, \\ Chengda Dong ${ }^{1}$, Huishan Shi ${ }^{1}$, Zhaojun Yan $^{2}$
}

${ }^{1}$ First Clinical Medical College, Shandong University of Traditional Chinese Medicine, Jinan, China; ${ }^{2}$ Department of Psychosomatic Medicine, Affiliated Hospital of Shandong University of Traditional Chinese Medicine, Jinan, China

Contributions: (I) Conception and design: Z Yan; (II) Administrative support: R Zhao; (III) Provision of study materials or patients: H Shi; (IV) Collection and assembly of data: A Song, J Liu, Q Chu; (V) Data analysis and interpretation: R Zhao, P Liu; (VI) Manuscript writing: All authors; (VII) Final approval of manuscript: All authors.

"These authors contributed equally to this work.

Correspondence to: Zhaojun Yan. Department of Psychosomatic Medicine, Affiliated Hospital of Shandong University of Traditional Chinese Medicine, No. 16369 Jingshi Road, Jinan 250014, China. Email: zr95510@163.com.

Background: Panic disorder (PD) is a kind of mental illness characterized by the symptom of recurring panic attacks. Qiangzhifang (QZF) is a novel decoction developed by Professor Zhaojun Yan based on a unique system of syndrome differentiation and clinical experience. It has achieved remarkable results after long-term clinical practice, but its mechanism of action is still unclear. This study aims to use network pharmacology and molecular docking to explore the mechanism of QZF in the treatment of PD.

Methods: We used the Traditional Chinese Medicine Systems Pharmacology Database and Analysis Platform (TCMSP), a literature search, and Encyclopedia of Traditional Chinese Medicine (ETCM) to find active ingredients and targets of QZF. We searched for PD targets in GeneCards, Online Mendelian Inheritance in Man (OMIM), the Comparative Toxicogenomics Database (CTD), and DrugBank. We established a PD target database, constructed a protein-protein interaction (PPI) network, and performed Gene Ontology (GO) and Kyoto Encyclopedia of Genes and Genomes (KEGG) enrichment analysis in order to screen possible pathways of action and analyze the mechanism.

Results: This study identified 84 effective components of QZF, 691 potential targets, 357 PD targets, and 97 intersectional targets. Enrichment analysis using the Database for Annotation, Visualization, and Integrated Discovery (DAVID) showed that QZF was associated with 118 biological processes (BPs), 18 cellular components (CCs), 35 molecular functions (MFs) [false discovery rate (FDR) <0.01], and 62 pathways (FDR <0.01). QZF mainly acts on its targets $A K T 1, F O S$, and APP through active ingredients such as quercetin, $\beta$-sitosterol, 4-(4'-hydroxybenzyloxy)benzyl methyl ether, harmine, 1,7-dimethoxyxanthone, and 1-hydroxy-3,7-dimethoxyxanthone to regulate serotonin, gamma-aminobutyric acid (GABA), cyclic adenosine monophosphate (cAMP), and other signal pathways to treat PD.

Conclusions: Through network pharmacology and molecular docking technology, we predicted the possible mechanism of $\mathrm{QZF}$ in the treatment of $\mathrm{PD}$, revealed the interaction targets and potential value of QZF, and provided a basis for its clinical application.

Keywords: Qiangzhifang (QZF); network pharmacology; molecular docking; panic disorder (PD)

Submitted Jun 08, 2021. Accepted for publication Aug 16, 2021.

doi: 10.21037/atm-21-4090

View this article at: https://dx.doi.org/10.21037/atm-21-4090 


\section{Introduction}

Panic disorder (PD) is a common mental illness, also known as acute anxiety attack, which refers to an anxiety disorder in which unexpected panic attacks occur repeatedly. It is clinically characterized by repeated and sudden strong fear, panic, or discomfort and may bring a sense of imminent death or loss of control. PD may manifest as different symptoms such as the cardiovascular system, respiratory system, digestive system, and nervous system (1). The prevalence rate of this disease is $3.6-5.1 \%$. It is more common among young and middle-aged people and more common in women than men. It can easily transform into chronic or even permanent disease if it is not diagnosed and treated in time. Its lifetime prevalence is $3.4-4.7 \%$ (2). Prolonged unhealed PD will not only seriously affect the quality of life and social function of its sufferers but even increase the chance of lifelong suicide attempts (3). Therefore, PD should be detected and treated as soon as possible to avoid further detriments to the patient's physical and mental health.

In 2019, novel coronavirus (COVID-19) rapidly spread around the world. The disease was highly infectious, fast transmission, high mortality and poor prognosis, affecting human economic, social and health, causing fear, anxiety and avoidance, and emphasizing the fear of patients with mental illness (4). Traditional Chinese medicine plays an important role in the prevention and control of the epidemic, and has unique advantages in the treatment of fear and anxiety, and other emotional diseases (5).

PD is treated mostly with selective serotonin reuptake inhibitors (SSRIs), benzodiazepine drugs (BZDs) and antidepressants tricyclic antidepressants (TCAs). Although these drugs can relieve panic attacks and improve anxiety, long-term use of these drugs is prone to adverse reactions, such as biological dependence, withdrawal reactions, and gastrointestinal discomfort (6). Therefore, there is an urgent need for safe and effective treatment methods with less side effects. Traditional Chinese medicines have the advantages of multiple components and multiple targets acting in coordination and fewer adverse reactions.

Professor Zhaojun Yan of Shandong Provincial Hospital of Traditional Chinese Medicine has created a unique system of syndrome differentiation for the treatment of mental and behavioral diseases, which has a profound clinical foundation. Qiangzhifang (QZF), invented under the theoretical guidance of Professor Zhaojun Yan, is composed of Morinda officinalis (ba ji tian, BJT), Pinellia (banxia, BX), jujube (da zao, DZ), Poria (fu ling, FL), Chinese yam (shan yao, SY),
Gastrodia (tian ma, TM), and Polygala (yuan zhi, YZ). Longterm clinical application has verified its definite curative effect in the treatment of fear, timidity, anxiety, compulsion, insomnia, and restlessness. However, the mechanism of QZF's therapeutic effect remains to be elucidated.

In recent years, network pharmacology has been increasingly applied in the research of traditional Chinese medicine. The comprehensive, systematic and holistic concept of network pharmacology is consistent with the characteristics of traditional Chinese medicine prescription multiple compound, multi-target point and multiple route, and its methods can clearly clarify the molecular mechanism of traditional Chinese medicine. The discovery of new targets of traditional Chinese medicine is a key breakthrough in drug development, and network pharmacology is expected to become the key direction of the research of traditional Chinese medicine prescription agents (7).

This study aims to analyze the multicomponent, multitarget, and multipath mechanism of QZF in the treatment of PD through network pharmacology techniques, in order to reveal the mechanism and potential clinical value of QZF and provide a basis for further in-depth research.

\section{Methods}

\section{Schematic diagram}

Figure 1 is a schematic diagram of the research methods. We used network pharmacology and molecular docking methods to predict the mechanism of action of QZF in the treatment of PD.

\section{Screening the main active ingredients and targets of $Q Z F$}

To mix QZF, compounds collected from BJT, BX, DZ, FL, and SY were derived from the Traditional Chinese Medicine Systems Pharmacology Database and Analysis Platform (TCMSP) (8). The names of the Chinese medicine components were used for the query. Oral availability (OB) and drug-like properties (DL) were included as the screening conditions. OB and DL are important indicators for evaluating pharmacokinetic absorption, distribution, metabolism, and excretion (ADME) (9). OB is the speed and extent to which the active ingredient or active group of the drug is absorbed into the systemic circulation; and DL indicates that the drug contains some specific functional groups or has the same or similar physical characteristics as most drugs (10). The screening value was set to $\mathrm{OB} \geq 30 \%$ and $\mathrm{DL} \geq 0.18$. We searched for the target proteins of each 


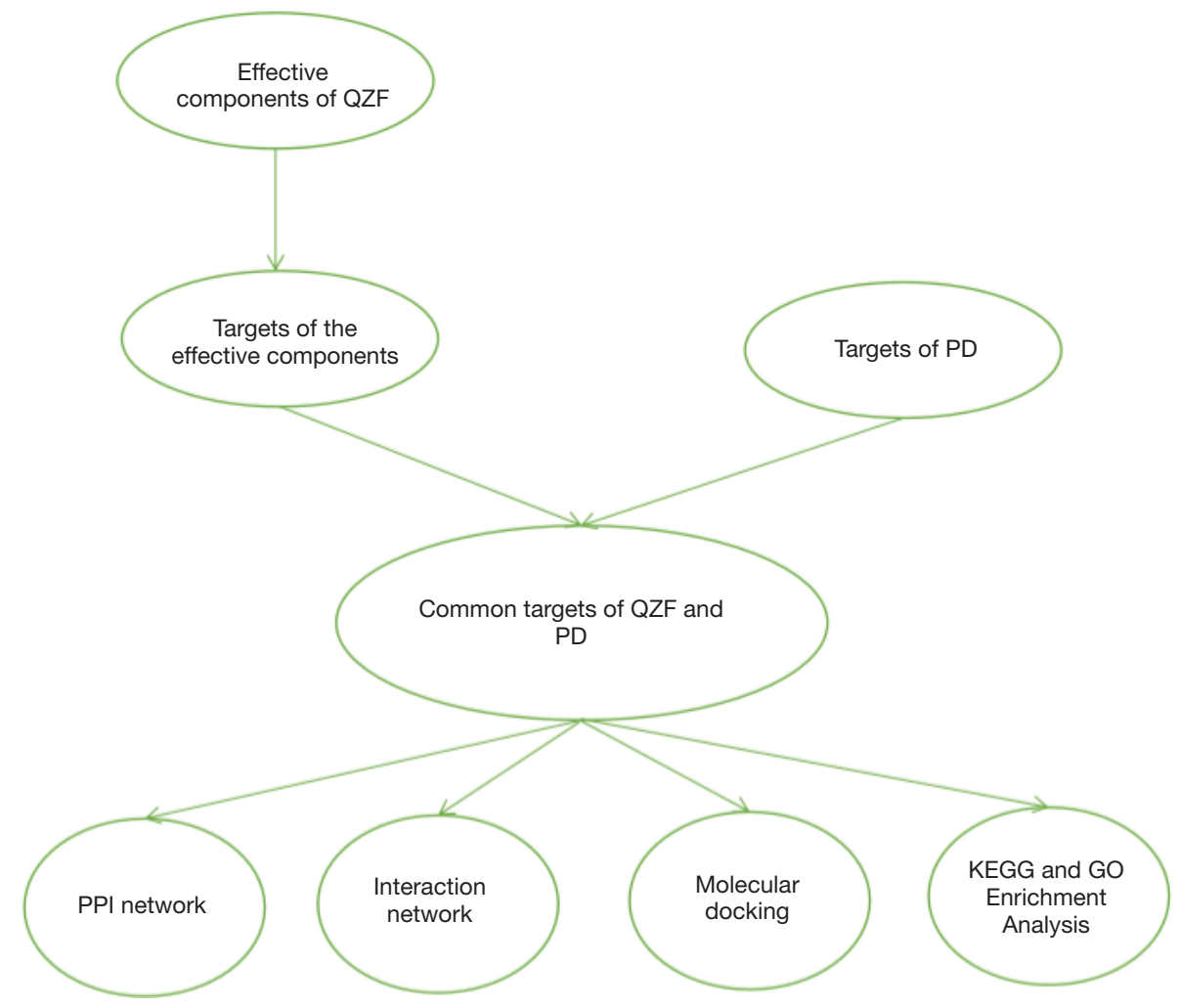

Figure 1 Figure 1 Flow chart of network pharmacology research on QZF in the treatment of PD. QZF, Qiangzhifang; PD, panic disorder; PPI, protein-protein interaction; KEGG, Kyoto Encyclopedia of Genes and Genomes; GO, Gene Ontology.

molecule in the "related targets" section and imported the results of the active ingredients and target information into WPS Office Excel tables. The UniProt protein database (11) was used to convert the target proteins into the corresponding gene names.

The TM and YZ compounds were collected from a literature search and query of the Encyclopedia of Traditional Chinese Medicine (ETCM) (12). The structure names of the collected compounds were searched in the PubChem database (13), and the chemical structure diagrams of each chemical component were summarized. The obtained 2D structural formula was uploaded to the Swiss ADME database (14) for screening, and the screening parameters were set with gastrointestinal absorption at "high". The 2D structure formula of the compound after screening was imported into the Swiss TargetPrediction database (14) to predict the target sites, and the output was summarized.

\section{PD disease target collection}

We searched the GeneCards database (15), Online
Mendelian Inheritance in Man (OMIM) database (16), Comparative Toxicogenomics Database (CTD) (17), and DrugBank database (18) with "panic disorder" as a keyword, removing duplicate targets, to establish the disease target data.

\section{Construction of protein-protein interaction (PPI) network}

We compared the target information of QZF and PD and used bioinformatics software (http://www.bioinformatics.com.cn/) to obtain the overlapping genes. The potential targets were imported into the String database (19). Cytoscape software (20) was used to draw the PPI network and calculate the degree value of each node of the PPI network in order to identify the core targets for the treatment of PD by QZF.

\section{Construction of QZF component-PD-target interaction network}

Cytoscape software (20) was used to draw the QZF component-PD-target interaction network. 


\section{Molecular docking verification}

We queried the PubChem website (13) to obtain the SDF structure files of the compounds. We then used Open Babel 2.3.2 software (21) to convert the SDF file into a PDB file and retrieved the key targets from the Protein Data Bank (22). PYMOL 2.3.4 software (23) was used to perform operations such as dehydration and ligand removal on the receptor proteins, and AutoDockTools software (24) was used to modify the receptor proteins, such as in their hydrogenation and charge balance. The Grid Box command under the Grid program was used to open the Grid Option tool to process the receptor protein, AutoDock Vina 1.1.2 (25) was used to do molecular docking of receptor proteins and smallmolecule ligands, and finally PYMOL software was used to visually analyze the docking results.

\section{Pathway enrichment analysis}

The potential targets were imported into the Database for Annotation, Visualization, and Integrated Discovery (DAVID) (26), "Homo sapiens" was selected as the species, and KEGG-pathway, GOTERM_BP_DIRECT(BP), GOTERM_CC_DIRECT(CC), and GOTERM_MF_ DIRECT(MF) were selected to perform Gene Ontology (GO) and Kyoto Encyclopedia of Genes and Genomes (KEGG) enrichment analysis of the overlapping genes. We obtained the basic data information about the biological processes (BPs), molecular functions (MFs), and cellular components (CCs) and about the involved pathways to draw GO histograms and KEGG enrichment bubble charts (http://www.bioinformatics.com.cn/).

\section{Statistical analysis}

Using Windows, WPS Office version 11.1.0.10667-Release for Windows version summary data, protein reciprocal PPI data were analyzed by version Cytoscape 3.7.2, and proteinprocessed data use AutoDock Vina 1.1.2 for molecular docking. The pathway enrichment visualization analysis is derived from the Database (DAVID) version 6.8. All relevant data analysis results are listed in the Results section below.

\section{Results}

\section{Screening the active ingredients and targets of $Q Z F$}

We input BJT, BX, DZ, FL, and SY into the TCMSP database, setting the parameters to $\mathrm{OB} \geq 30 \%$ and $\mathrm{DL}$ $\geq 0.18$. The SwissTargetPrediction database was used to predict the target points of TM and YZ, and the setting of "high" gastrointestinal absorption was chosen. The obtained active ingredients numbered 16 for BJT, 11 for BX, 18 for DZ, six for FL, 12 for SY, 12 for TM, and 13 for YZ. A total of 88 compounds were obtained, but after removing duplicate values, 84 remained (Tables 1,2). The 84 compounds were further studied using the UniProt database and Swiss TargetPrediction database, which yielded a total of 691 target genes of them.

\section{Identification of disease targets}

A total of 133 PD disease targets were collected through the GeneCards website, 118 PD targets were collected through the OMIM website, 195 PD disease targets were collected through the CTD website, and $80 \mathrm{PD}$ disease targets were collected through the DrugBank website. This total of 526 PD targets became 357 after removing duplicates.

\section{Construction of the PPI network}

We intersected the targets of the QZF components with the PD disease targets by using bioinformatics software (http://www.bioinformatics.com.cn/). As a result, a total of 97 common targets were obtained (Figure 2). We used Cytoscape software to visualize the PPI network. After discarding unmatched targets, 95 remained (Figure 3). We obtained a total of 95 nodes and 912 edges. The size and the lightness of the color of the node indicate the node degree (number of connections to it), and the thickness and the lightness of the color of each edge indicate the strength of that interaction. The top 10 targets according to node degree were serine/threonine kinase 1 (AKT1), the proto-oncogene (FOS), amyloid beta precursor protein (APP), sodium-dependent serotonin transporter (SLC6A4), catechol O-methyltransferase (COMT), interleukin-6 (IL-6), cell tumor antigen $\mathrm{p} 53$, a caspase (CASP3), vascular endothelial growth factor A (VEGFA), and tumor necrosis factor (TNF) (Figure 4).

\section{QZF active ingredient-PD-target interaction network construction}

We obtained 155 nodes and 552 edges using Cytoscape software for analysis. As shown in Figure 5, pink indicates the drug QZF, red indicates the disease PD, green indicates the active ingredients of QZF, and purple indicates the common targets of QZF and PD (Figure 5). 
Table 1 Active ingredients and targets of QZF

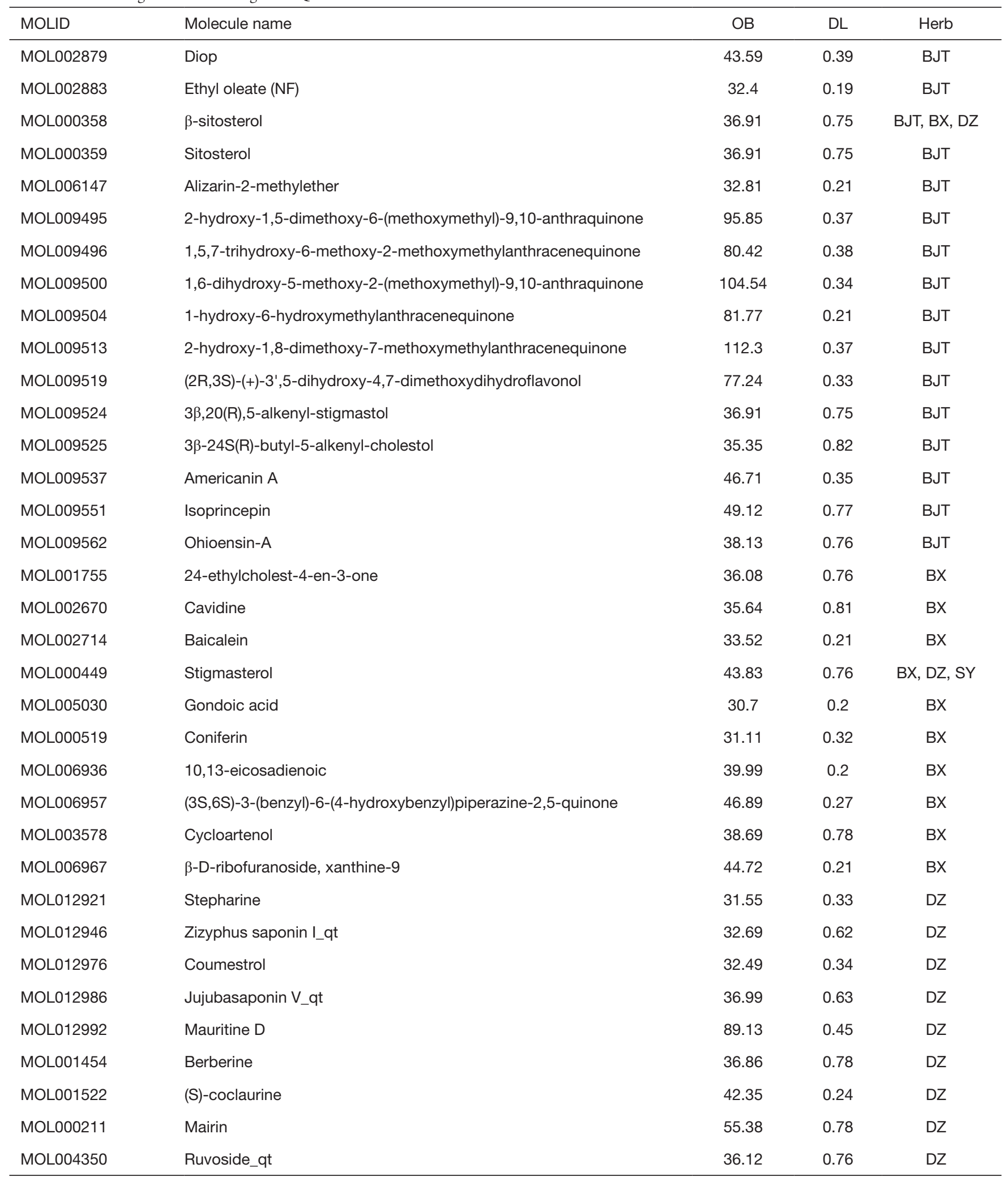

Table 1 (continued) 
Table 1 (continued)

\begin{tabular}{|c|c|c|c|c|}
\hline MOLID & Molecule name & OB & DL & Herb \\
\hline MOL000627 & Stepholidine & 33.11 & 0.54 & DZ \\
\hline MOL007213 & Nuciferin & 34.43 & 0.4 & $\mathrm{DZ}$ \\
\hline MOL000787 & Fumarine & 59.26 & 0.83 & $\mathrm{DZ}$ \\
\hline MOL000096 & $(-)$-catechin & 49.68 & 0.24 & $\mathrm{DZ}$ \\
\hline MOL000098 & Quercetin & 46.43 & 0.28 & DZ \\
\hline MOL000273 & $\begin{array}{l}\text { (2R)-2-[(3S,5R,10S,13R,14R,16R,17R)-3,16-dihydroxy-4,4,10,13,14- } \\
\text { pentamethyl-2,3,5,6,12,15,16,17-octahydro-1H-cyclopenta[a] } \\
\text { phenanthren-17-yl]-6-methylhept-5-enoic acid }\end{array}$ & 30.93 & 0.81 & $\mathrm{FL}$ \\
\hline MOL000282 & Ergosta-7,22E-dien-3beta-ol & 43.51 & 0.72 & $\mathrm{FL}$ \\
\hline MOL000283 & Ergosterol peroxide & 40.36 & 0.81 & FL \\
\hline MOL000296 & Hederagenin & 36.91 & 0.75 & $\mathrm{FL}$ \\
\hline MOL001559 & piperlonguminine & 30.71 & 0.18 & SY \\
\hline MOL001736 & (-)-taxifolin & 60.51 & 0.27 & SY \\
\hline MOL000322 & Kadsurenone & 54.72 & 0.38 & SY \\
\hline MOL005430 & Hancinone C & 59.05 & 0.39 & SY \\
\hline MOL005465 & AIDS180907 & 45.33 & 0.77 & SY \\
\hline MOL000953 & CLR & 37.87 & 0.68 & SY \\
\hline
\end{tabular}

QZF, Qiangzhifang; OB, oral availability; DL, drug-like properties; BJT, Morinda officinalis (ba ji tian); BX, Pinellia (banxia); DZ, jujube (da zao); SY, Chinese yam (shan yao); FL, Poria (fu ling).

\section{Molecular docking}

Quercetin, beta-sitosterol, 4-(4'-hydroxybenzyloxy)benzyl methyl ether, harmine, 1,7-dimethoxyxanthone, and 1-hydroxy-3,7-dimethoxyxanthone were the five active ingredients of QZF with the highest node degrees. We docked the main active ingredients of QZF with the top three core targets $A K T 1$, Fos, and APP one by one (Table 3).

The more stable the binding conformation, the lower the required binding energy. Binding energy $\leq-4.25 \mathrm{kcal} / \mathrm{mol}$ indicates weaker binding activity, $\leq-5.0 \mathrm{kcal} / \mathrm{mol}$ indicates good binding activity, and $\leq-7.0 \mathrm{kcal} / \mathrm{mol}$ indicates strong binding activity. In this study, binding energy $\leq-8.5 \mathrm{kcal} / \mathrm{mol}$ was chosen as the screening condition, and PYMOL software was used to draw a $3 \mathrm{D}$ docking schematic diagram of the docking results. We investigated the binding mode between receptor protein $A K T 1$ and the small-molecule ligand beta-sitosterol and that between receptor protein $A K T 1$ and the small-molecule ligand quercetin. Figure 6 shows the binding mode between the receptor protein 
Table 2 Active ingredients and targets of QZF (YZ and TM)

\begin{tabular}{|c|c|c|c|c|}
\hline ID & Molecule name & Pharmacokinetics (Gl absorption) & Drug likeness & Herb \\
\hline TM2 & Cetylic acid, hexadecanoic acid, palmitic acid & High & Yes & TM \\
\hline TM3 & p-hydroxybenzaldehyde & High & Yes & TM \\
\hline TM4 & Bis(4-hydroxybenzyl)ether & High & Yes & TM \\
\hline TM6 & 4,4'-dihydroxydiphenyl methane & High & Yes & TM \\
\hline TM7 & 4-ethoxymethylphenyl-4'-hydroxybenzylether & High & Yes & TM \\
\hline TM8 & Gastrodamine & High & Yes & TM \\
\hline TM9 & 4-hydroxybenzyl alcohol & High & Yes & TM \\
\hline TM12 & 4-(4'-hydroxybenzyloxy)Benzyl methyl ether & High & Yes & TM \\
\hline YZ1 & Harman & High & Yes & $\mathrm{YZ}$ \\
\hline YZ2 & Onjixanthone I & High & Yes & $\mathrm{YZ}$ \\
\hline YZ3 & Î'-carboline-1-carboxylic acid, methyl ester & High & Yes & $\mathrm{YZ}$ \\
\hline YZ4 & 1-ethoxycarbonyl-beta-carboline & High & Yes & $\mathrm{YZ}$ \\
\hline YZ5 & 1,7-dimethoxyxanthone & High & Yes & $\mathrm{YZ}$ \\
\hline YZ6 & 1-hydroxy-3,7-dimethoxyxanthone & High & Yes & $\mathrm{YZ}$ \\
\hline YZ7 & 1-hydroxy-3,6,7-trimethoxy xanthone & High & Yes & $\mathrm{YZ}$ \\
\hline YZ13 & Harmine & High & Yes & YZ \\
\hline
\end{tabular}

QZF, Qiangzhifang; GI, gastrointestinal; TM, Gastrodia (tian ma); YZ, Polygala (yuan zhi).

AKT1 and the small-molecule ligand beta-sitosterol. The amino acid residues Ala230 and Glu228 formed a hydrogen bond with the small-molecule ligand beta-sitosterol. The amino acid residues Tyr229, Ala177, Val164, Lys179, Gly159, Gly162, Leu181, Thr160, Asp292, Glu278, Thr291, and Met281 formed hydrophobic interactions with the small-molecule ligand beta-sitosterol. Figure 7 shows the binding mode between the receptor protein $A K T 1$ and the small-molecule ligand quercetin. The amino acid residues Lys179, Glu228, and Ala230 formed hydrogen bonds with the small-molecule ligand quercetin. The amino acid residues Tyr229, Phe438, Ala177, Leu156, Val164, Gly159, Asp292, Met227, and Met281 formed hydrophobic interactions with the small-molecule ligand quercetin. Taken together, these data suggest that quercetin and betasitosterol may be the key active ingredients of QZF in the treatment of PD.

\section{GO function enrichment analysis}

GO analysis showed that the 95 targets of QZF in the treatment of PD mainly involved $456 \mathrm{BPs}, 55 \mathrm{CCs}$, and 


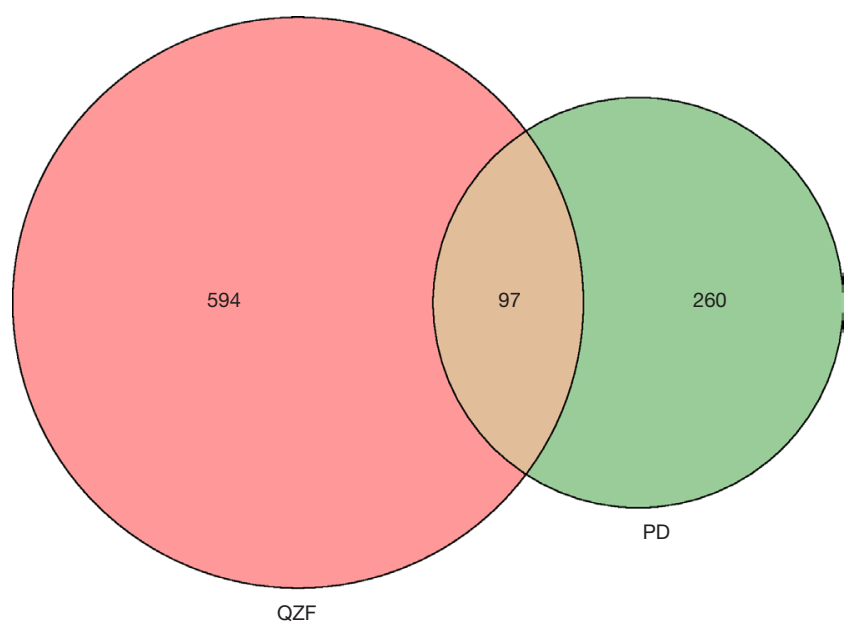

Figure 2 Venn diagram of the common targets beween QZF and PD. QZF, Qiangzhifang; PD, panic disorder.

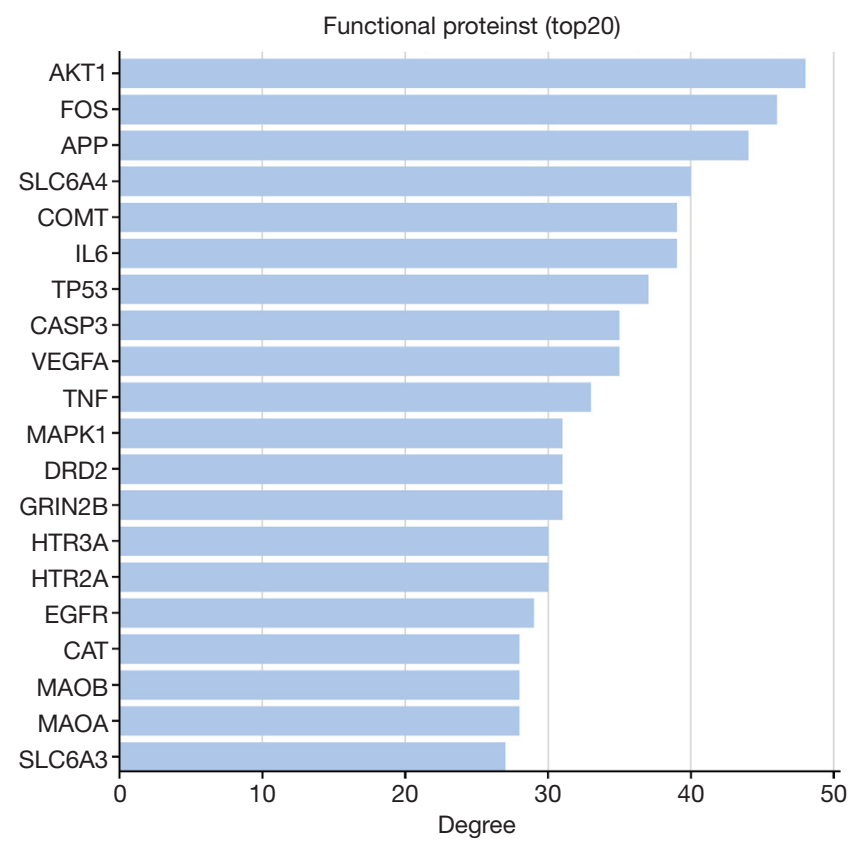

Figure 4 Top 20 protein targets.

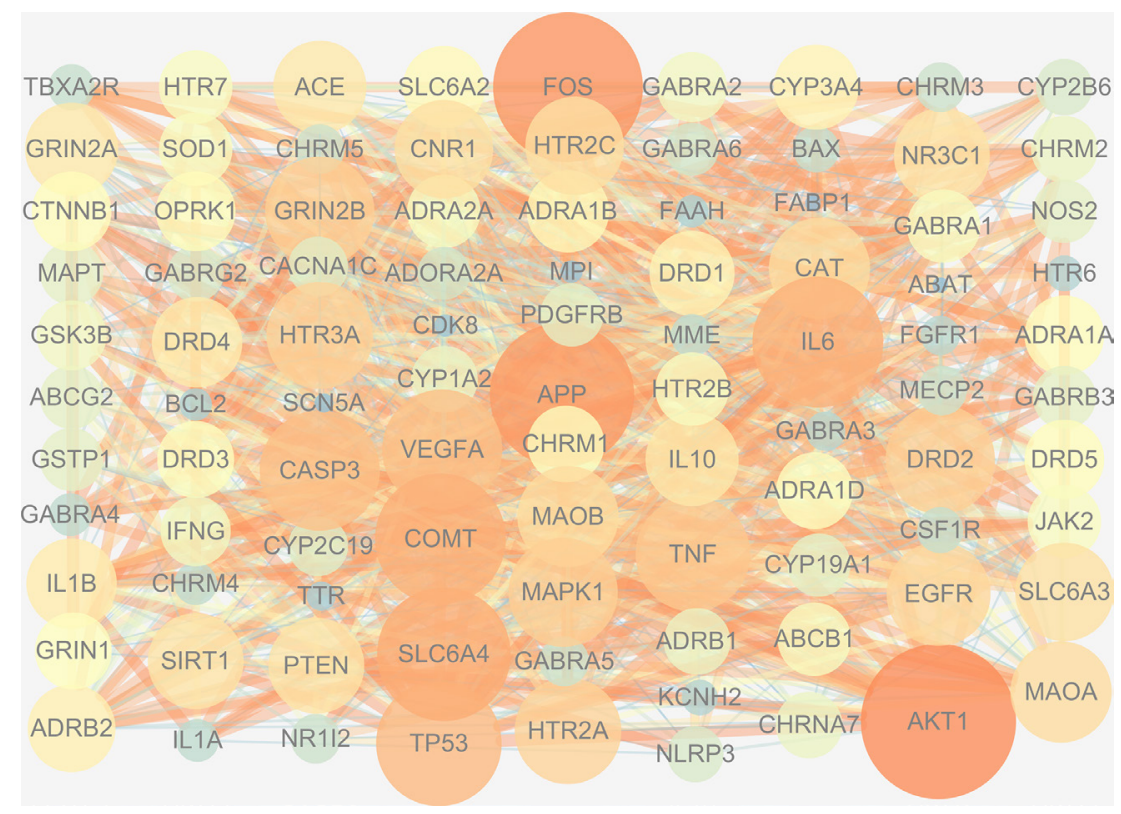

Figure 3 PPI network of QZF and PD. PPI, protein-protein interaction; QZF, Qiangzhifang; PD, panic disorder.

79 MFs. With $\mathrm{P}<0.01$ and false discovery rate $(\mathrm{FDR})<0.01$ as the screening criteria, a total of $118 \mathrm{BPs}, 18 \mathrm{CCs}$, and $35 \mathrm{MFs}$ were obtained. We sorted the corrected $\mathrm{P}$ values from small to large, and the top 10 targets by $\mathrm{P}$ value were analyzed (Figure 8). The BPs of the targets included response to drug, positive regulation of cell proliferation, adenylate cyclase-activating adrenergic receptor signaling pathway, synaptic transmission, and cholinergic synaptic transmission. The CCs included integral component of plasma membrane, postsynaptic membrane, plasma 


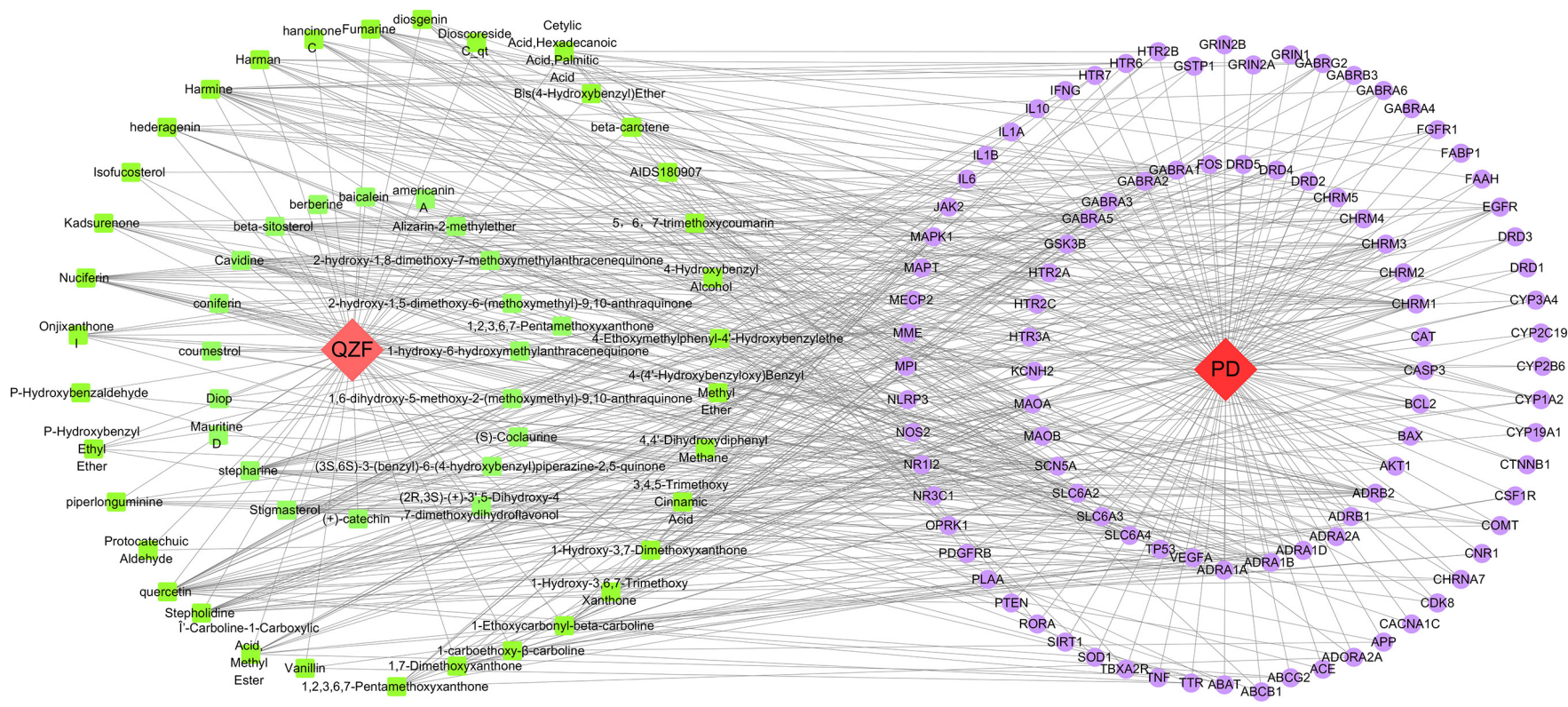

Figure 5 QZF component-PD-target interaction network. QZF, Qiangzhifang; PD, panic disorder.

Table 3 Docking results between the main active ingredients and core targets

\begin{tabular}{|c|c|c|c|}
\hline Active compound & Target name & PDB ID & Docking score $(\mathrm{kcal} / \mathrm{mol})$ \\
\hline \multirow[t]{2}{*}{ 1,7-dimethoxyxanthone } & $A K T 1$ & $4 E K L$ & -8.0 \\
\hline & $A P P$ & $1 A A P$ & -5.5 \\
\hline \multirow[t]{2}{*}{ 1-hydroxy-3,7-dimethoxyxanthone } & AKT1 & $4 E K L$ & -7.7 \\
\hline & $A P P$ & $1 A A P$ & -5.6 \\
\hline \multirow[t]{3}{*}{ 4-(4'-hydroxybenzyloxy)benzyl methyl ether } & AKT1 & $4 E K L$ & -7.3 \\
\hline & $A P P$ & $1 A A P$ & -5.1 \\
\hline & FOS & $1 F O S$ & -4.8 \\
\hline Beta-sitosterol & AKT1 & $4 E K L$ & -9.0 \\
\hline \multirow[t]{3}{*}{ Harmine } & AKT1 & $4 E K L$ & -7.7 \\
\hline & $A P P$ & $1 A A P$ & -4.9 \\
\hline & FOS & $1 F O S$ & -4.7 \\
\hline \multirow[t]{3}{*}{ Paroxetine } & AKT1 & $4 E K L$ & -8.4 \\
\hline & $A P P$ & $1 A A P$ & -4.9 \\
\hline & FOS & $1 F O S$ & -5.5 \\
\hline \multirow[t]{2}{*}{ Quercetin } & $A K T 1$ & $4 E K L$ & -8.5 \\
\hline & $A P P$ & $1 A A P$ & -6.2 \\
\hline
\end{tabular}




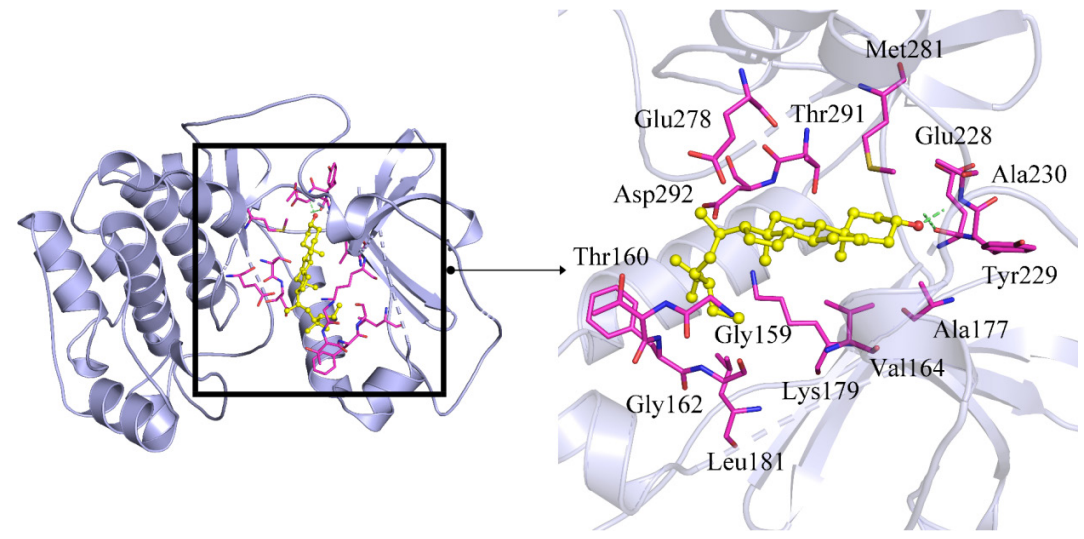

Figure 6 AKT1 and $\beta$-sitosterol.
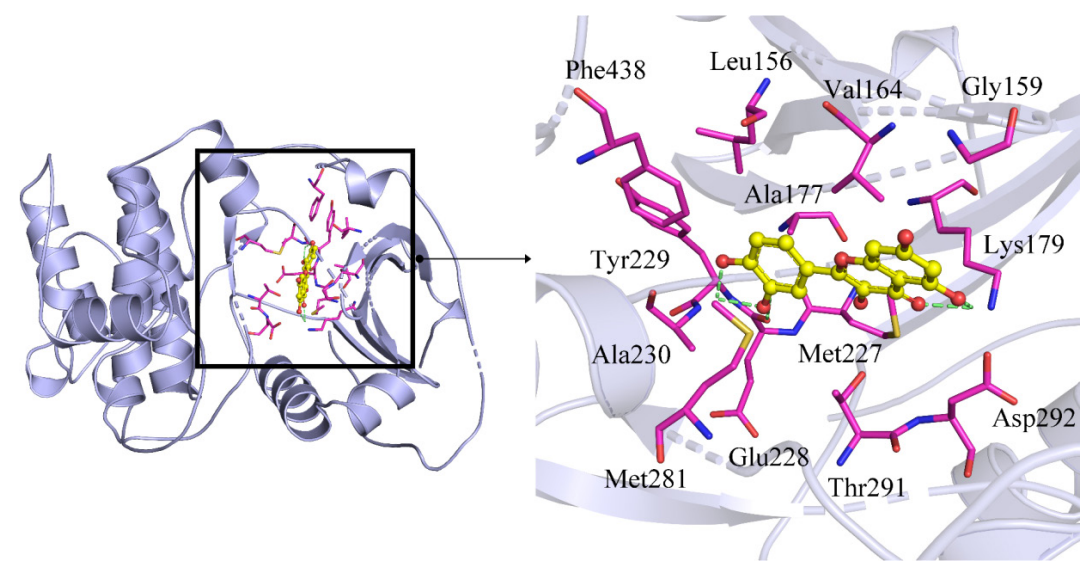

Figure 7 AKT1 and quercetin.

membrane, synapse, and cell junction. The MFs included drug binding, enzyme binding, identical protein binding, extracellular ligand-gated ion channel activity, and dopamine binding.

\section{KEGG analysis}

The obtained predicted targets were analyzed using DAVID software for the KEGG analysis, and a total of 97 pathways were obtained. Sixty-two pathways had a $P$ value $<0.01$, as the screening criterion, and the top 20 signal pathways in the KEGG enrichment pathway analysis based on the corrected $\mathrm{P}$ value are shown in Figure 9. Each pathway contained different targets (Table 4). Therefore, we speculate that QZF in the treatment of PD mainly acts through neuroactive ligand-receptor interactions, calcium signaling, serotonergic synapses, dopaminergic synapses, cyclic adenosine monophosphate (cAMP) signaling, cholinergic synapses, and GABAergic synapses.

\section{Discussion}

$\mathrm{PD}$, also known as acute anxiety attack, is a subtype of anxiety disorder. Its symptoms include heart palpitations, chest discomfort, rapid breathing, trembling, nausea, and dizziness, accompanied by a strong sense of imminent death or loss of control. Its onset is sudden, its manifestations last for several or tens of minutes, and it is self-limiting. Modern medicine holds that its pathogenesis is affected by a variety of mechanisms [including genetic factors and the neurotransmitters 5-hydroxytryptamine (5-HT; serotonin), norepinephrine, and gamma-aminobutyric acid (GABA)] (27).

QZF is a novel decoction developed by Professor Zhaojun Yan. The decoction has components of seven sources, BJT, BX, DZ, FL, SY, TM, and YZ. All these medicinal sources have the functions of strengthening 

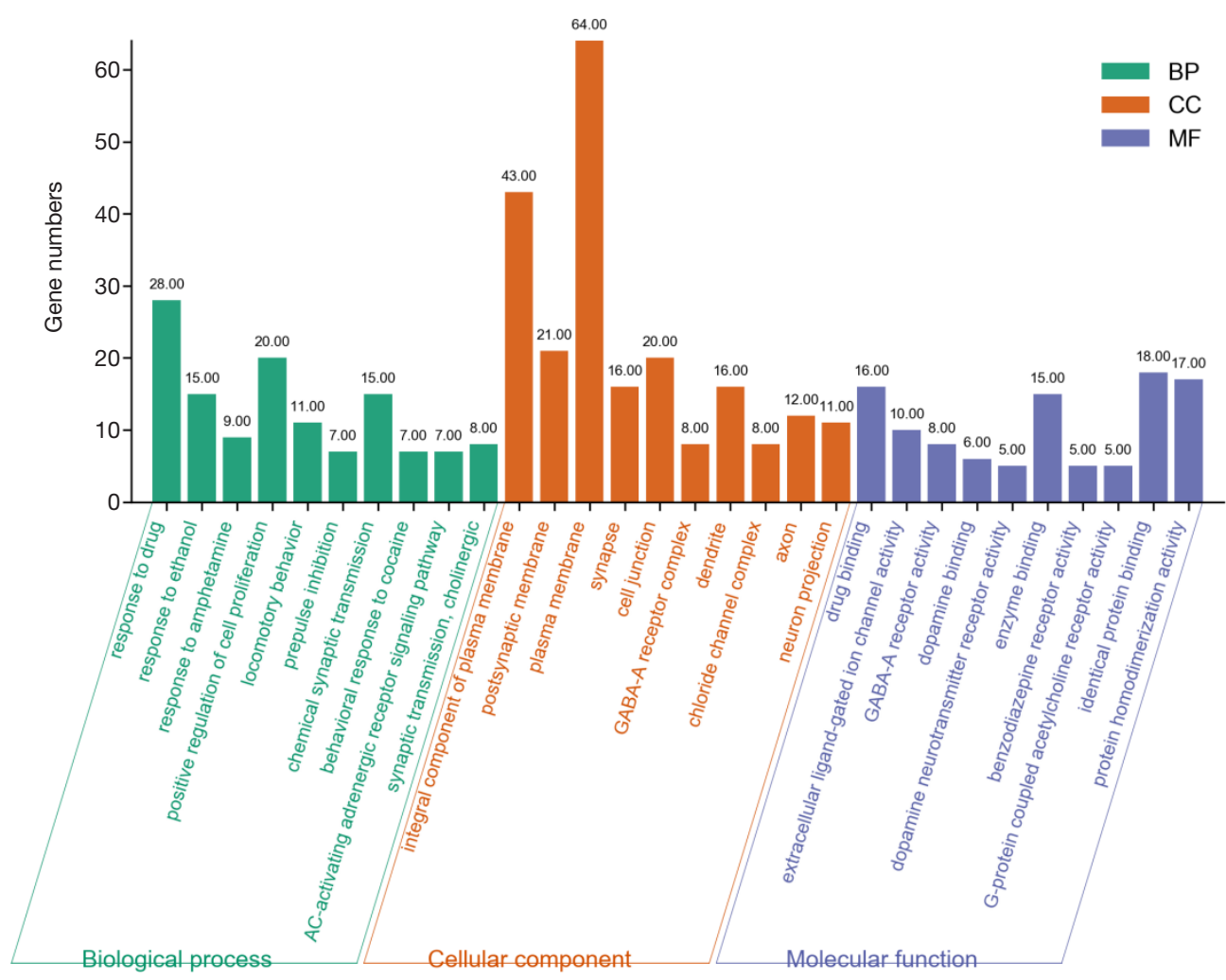

Figure 8 Histogram for the GO enrichment analysis of common targets. GO, Gene Ontology; BP, biological process; CC, cellular component; MF, molecular function.

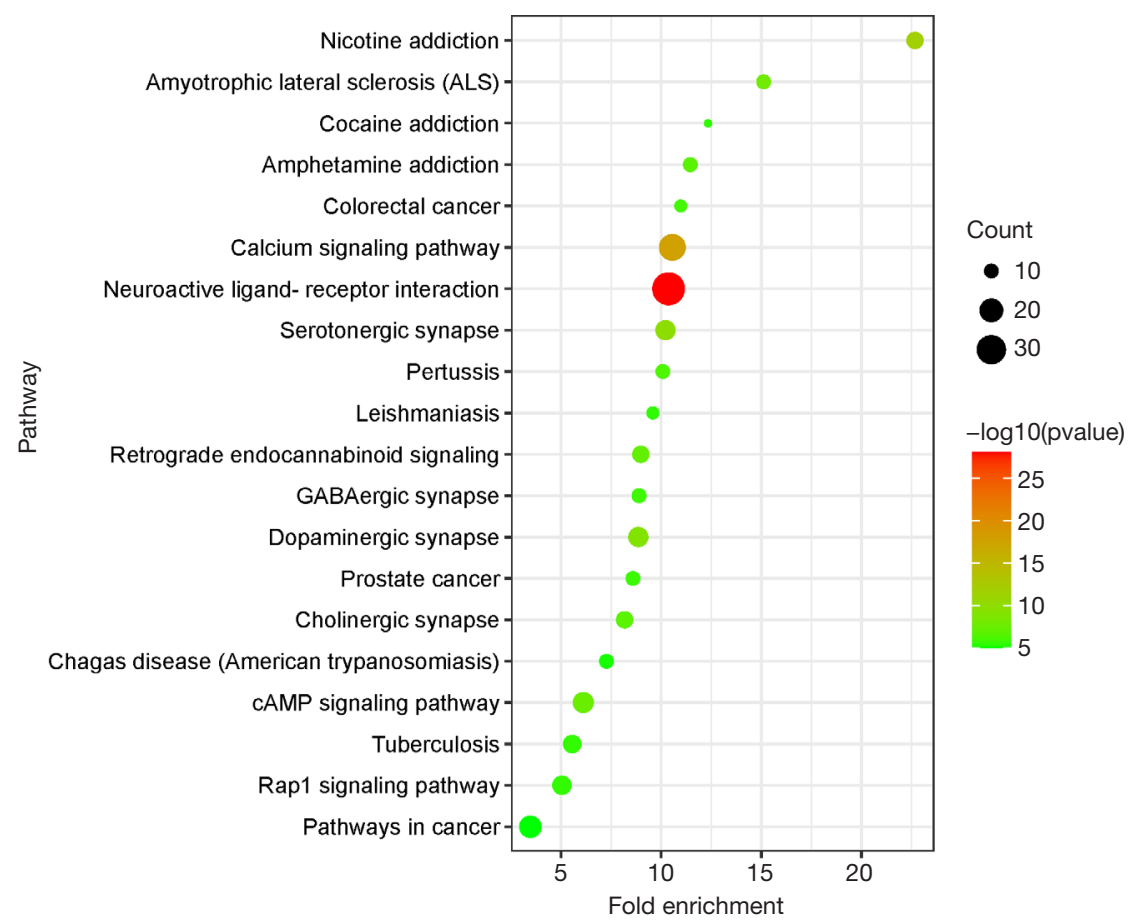

Figure 9 Bubble plots of the top 20 pathways. 
Table 4 KEGG analysis of the top 20 pathways

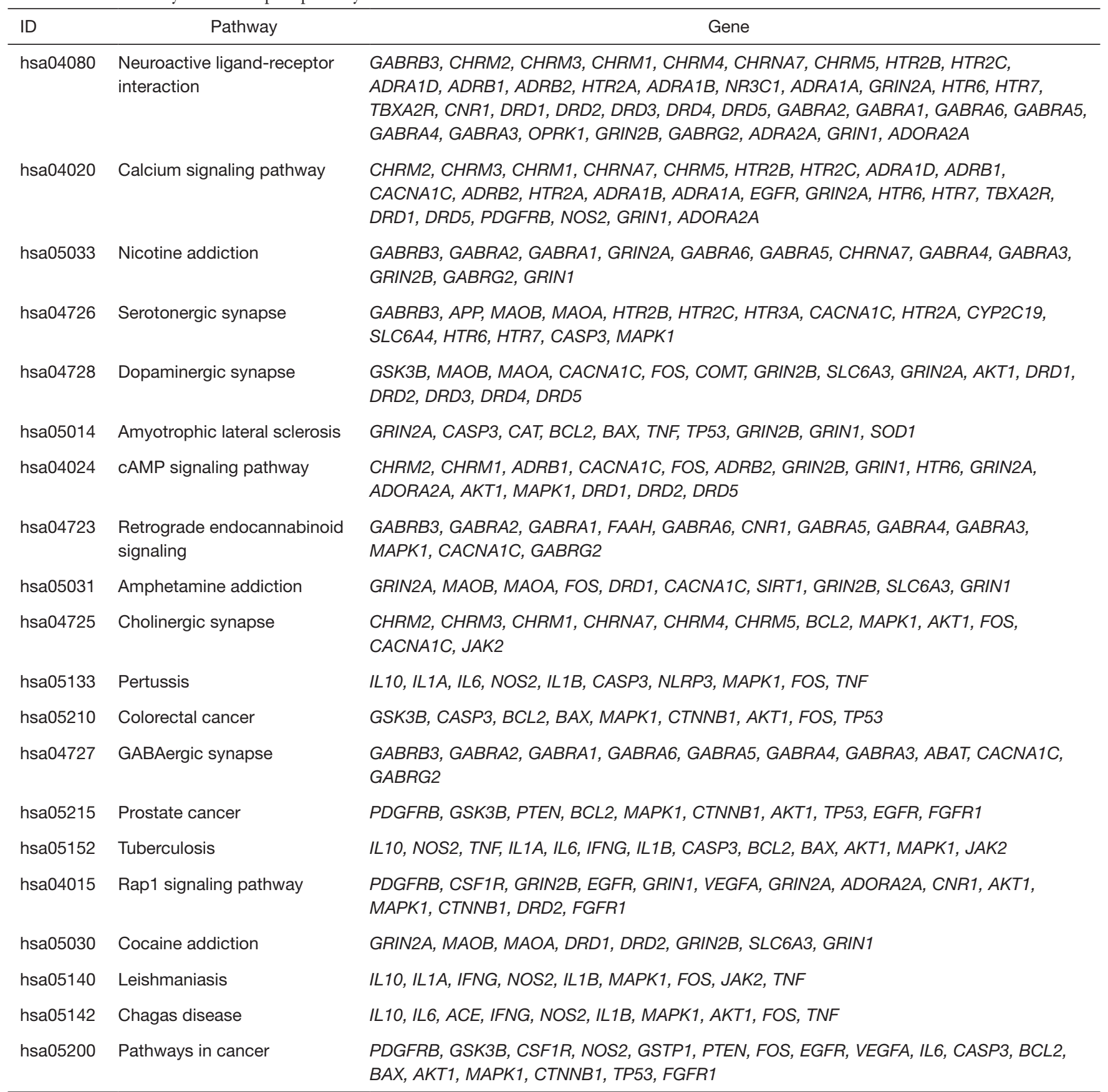

KEGG, Kyoto Encyclopedia of Genes and Genomes.

the mind, relieving shock, and nourishing and improving the mind. After long-term clinical practice, it has been found that QZF decoction can effectively alleviate fearful behaviors in patients. It is shown that Inulin starch Morinda officinalis oligosaccharide (IOMO) can shorten the freezing time of shock rats, inhibit fear behavior, and improve anxiety-like behavior (28). Ganmai jujube soup (jujube, licorice, wheat), and Pinellia thick Pu soup (Pinellia, thick $\mathrm{Pu}$, Poria cocos, ginger, Su leaf) two classic prescription are two important prescriptions for the treatment of emotional disease, among which Banellia, jujube, porkahoe, widely used to treat anxiety, fear, depression and other diseases. 
Yam can delay the aging process of the tissue structure of the immune organs, protect the tissue structure of the thymus and spleen of the important immune organs in mice, and have the role of immune regulation and treatment of infectious diseases (29). The gastrodia elata extract extends the time on the open arm and enters the percentage of the open arm, increasing the exploratory behavior of rats and plays an anti-anxiety role by regulating the GABA neurotransmitter system (30). The distant active ingredient 3,6'-diaphophol sucrose (DISS) increases the acidification level of cyclic phosphoradenosine reaction element binding protein [CAMP-response element binding protein (CREB)] in the hippocampus of chronic stressed rats, promote transcription of brain-derived neurotrophic factor (BDNF), and then protect neurons and improve depression and stress states (31). Therefore, drug combination can not only improve fear and anxiety symptoms, but also have an antiinflammatory and immune effect. We found that the total time of freezing posture (such as: static, curl, stagnation, slight swing movement, etc.) was commonly used to detect the fear behavior in rats. Compared with the model group, the freezing time was significantly shortened and dopamine (DA) and tyrosine hydrohydroxylase (TH) content in brain tissue was reduced, we believed that the TH mRNA expression reduced $\mathrm{TH}$ content, thus affecting the DA content and thus improving the extraction composite fear behavior in rats (32). It is shown that the cAMP-PKA pathway affects $\mathrm{TH}$ acidification, and PKA acts on TH residues to phosphorylation and participates in short-and long-period regulation of TH, leading to an increase in DA synthesis. Therefore, we speculate that QZF intervention in the cAMP-PKA signaling pathway for the treatment of PD (33-35).

This study predicts the potential action mechanism of QZF for PD through network pharmacology and molecular docking methods. First, the screening of QZF active components and action targets revealed that 84 active components, 691 target genes. The PD targets are also collected. Then, the PPI network visualization of the obtained QZF and PD intersection genes was performed and arranged the targets according to the size of the degree value, which showed a total of 95 potential targets for QZF treating PD. Molecular docking shows that the main active components in QZF and the core target have good binding activity, and performs GO enrichment analysis and KEGG pathway analysis on the potential targets, which reflects the multi-component, multi-target and multiple pass complex characteristics of QZF in treating PD.
Figure 1 depicts the drug-component-target relationships, showing that the six ingredients with the highest node degrees were the main active ingredients of quercetin, $\beta$-sitosterol, 4-(4'-hydroxybenzyloxy)benzyl methyl ether, harmine, 1,7-dimethoxyxanthone, and 1-hydroxy-3,7dimethoxyxanthone.Quercetin has antioxidant, antiinflammatory and neuroprotective effects. It can increase the mRNA level of BDNF and reduce that of inducible nitric oxide synthase, thereby improving anxiety-like behaviors caused by chronic neuroinflammation and reducing fear memory (36). $\beta$-sitosterol is a common component of BJT, $\mathrm{BX}$, and DZ. It is a plant sterol with anti-inflammatory, tumor-inhibiting, and antidepressant effects. It improves the anxiety symptoms of rats by regulating the gene expression of the $\mathrm{GABA}_{\mathrm{A}}$ signaling pathway (37). Harmine is a natural alkaloid with anti-inflammatory, antitumor, antianxiety, and immune-promoting effects. Harmine can enhance GABAergic transmission of neurons projecting to mouse basal amygdala by increasing the release of GABA at the presynaptic terminal to improve anxiety and depression (38).

We obtained 357 PD targets. PPI analysis revealed that there are 95 common targets between this Chinese medicine and this disease, with a total of 95 nodes and 912 edges. The top 10 targets are AKT1, FOS, APP, SLC6A4, COMT, IL6, $p 53, C A S P 3$, VEGFA, TNF. It is shown that $A K T$ (also known as protein kinase $\mathrm{B}$ or $\mathrm{PKB})$ contains three closely related isotypes $A K T 1, A K T 2$ and $A K t 3$ (39). $A K T 1$ are one of them, and that lack of $A K T 1$ increases the behavior of anxiety in mice. However, restoring the expression of AKT1 in the prefrontal cortex (PFC) can improve fear memory, behavior, where the GAGB pathway mediated this process (40). In addition to playing an important role in synaptic transmission, memory, and psychosis, $A K T$ is also one of the most common highly activated protein kinases in human cancer. Excessive activation of $A K T$ can also affect downstream effectors and mediate a variety of pathways conducive to tumorigenesis (39). FOS is closely associated with a variety of cancers, such as prostate cancer (41), ovarian cancer (42), breast cancer (43), etc. FOS deficiency in prostate cancer cell can increase cell proliferation and induce carcinogenic pathways changes, and the loss of FOS accelerates the progression of advanced latent prostate cancer disease (41). Lateral subnuclear FOS protein expression in the rat dorsal nucleus, GABA receptor antagonist (lwDR) increased significantly and activated GABAergic neurons, hydroxytonergic neurons in lwDR, causing intense panic escape behavior (44). 5-HT (2A) receptor antagonists and 5-HT (2C) agonists to 
weaken FOS expression in the dorolateral caudal thalamus $(\mathrm{CPu})(45)$. A trace infusion of TNF- $\alpha$ prevented c-FOS expression in the hippocampus and blocks fear memory and review (46). Fear memory induces the phosphorylation of the transcription factor CREB and regulates the expression of the early gene c-FOS. After fear behavior tests, CREB phosphorylation levels in the amygdala central nucleus and FOS immune response nucleus, and activation of CREB was involved in the formation of long-term fear memory (47). $A P P$ is a precursor protein from amyloid $\beta$-protein $(\mathrm{A} \beta)$, and studies suggest improved anxiety, depression behavior of amyloid $\beta$ in rats by upregulating the $\mathrm{BDNF} / \mathrm{trkB}$ pathway and inhibiting hippocampal autophagy (48). IL-6 is a multifunctional, multi-effect, and polytropic cytokine where PD patients are in proinflammatory states and whose severity is positively associated with $I L-6$ (49). Studies have shown that $I L-6$ plays an important role in local inflammation, is the most important carcinogenic factor and affects cell proliferation, metastasis and inflammation (50). $T N F$ is an anti-inflammatory cytokinine, widely involved in cell differentiation, apoptosis, and immune response, and plays an important role in autoimmune diseases and tumorigenic mechanisms (51,52). Studies have suggested that cAMP plays an important role in regulating TNFaexpression. For example, an increase in cell cAMP inhibited TNF- $\alpha$ production (53). TNF- $\alpha$ plays an important role in the central nervous system as an immune factor, giving multiple TNF-ato simulate chronic inflammatory response states can cause anxiety-like behavior and damage conditional fear memory (51).

Molecular docking further confirmed the screening results. The main active ingredients of quercetin, betasitosterol, 4-(4'-hydroxybenzyloxy)benzyl methyl ether, harmine, 1,7-dimethoxyxanthone, and 1-hydroxy-3,7dimethoxyxanthone have good binding activity with the potential targets $A K T 1, F O S$, and $A P P$, and the conformations of the binding complexes are stable, indicating that these are the key active ingredients and targets of QZF in the treatment of PD.

The GO function and KEGG pathway enrichment analyses of the key targets were carried out to further explore the mechanism of QZF in the treatment of PD. QZF may act through pathways involved in the response to drugs, positive regulation of cell proliferation, adenylate cyclase activation of the adrenergic receptor signaling pathway, synaptic transmission, and cholinergic synaptic transmission, and it may act at the plasma membrane, postsynaptic membrane, plasma membrane, synapse, and cell connections to facilitate drug binding, enzyme binding, identical protein binding, extracellular ligand-gated ion channel activity, and dopamine binding, and thereby regulate neuroactive ligand-receptor interactions, serotoninergic synapses, cAMP signaling, GABAergic synapses, and other pathways to improve panic and relieve anxiety.

\section{5-HT patbway}

The amygdala is a key brain area for fear processing. This brain area is regulated by the neurotransmitter serotonin. 5-HT neurons in the dorsal raphe nucleus regulate the basolateral amygdala (BLA) circuit by enhancing the release of 5-HT (54). Postplantar electric shock evaluation of mice with 5-HT synthesis deficiency (Tph2-deficient mice) found that the freezing time of mice increased, 5-HT was released rapidly in the amygdala, and the damage to GABAergic synaptic transmission significantly increased the level of c-FOS protein (55). The pairing of a conditioned stimulus and an unconditioned stimulus can enhance the release of 5-HT in the BLA and promote the expression of c-FOS in the dorsal raphe nucleus 5-HT cells, while the optogenetic excitation of 5-HT axons in the BLA may eliminate the effects of 5-HT release during the conditioned stimulus and unconditioned stimulus (56). 5-HTT is a transmembrane protein that controls the duration and degree of 5-HT neurotransmission by removing 5-HT from outside the cell. 5-HTT knockout (5-HTTKO) mice present significantly elevated extracellular 5-HT levels and an abnormal dendritic spine density of the main BLA neurons, which leads to the impairment of the fading of fear memory, and the time for the fading memory of fear is prolonged (57). However, excessive secretion of 5-HT may also lead to increased panic. Patients with anxiety who take 5-HT agonists orally and have acutely elevated 5-HT levels can have anxiety and panic attacks (58). SSRIs are the standard first-line drug treatment for PD (59). Short-term use of SSRIs can increase 5-HT levels and cortisol levels. Patients with PD are more anxious than controls (58), and acute medication with SSRIs will increase fear and panic, but chronic medication does not (54).

\section{GABA patbway}

The striatum contains many GABA neurons, and low GABAergic levels can cause panic. Microinjection of GABAergic antagonists into the striatum can cause defensive behavior in rats, showing freezing and evasion 
responses (60). Blocking $\mathrm{GABA}_{\mathrm{A}}$ receptors on the dorsal side of the midbrain can cause panic attacks, and neurons in the deep layer of the superior colliculus that excite the striatum and activate caudate putamen (CPU) play an important role in this process (61). The hippocampus is also involved in the process of panic attacks. In animal models of fear, the administration of tiagabine (GABA reuptake inhibitor) can significantly reduce hippocampal hyperexcitability and abnormal fear circuit activation (62). Stimulation of the BLA-dorsal periaqueductal gray can worsen paniclike attacks (63). If the number of $\mathrm{GABA}_{\mathrm{A}}$ receptors in the prefrontal cortex and hippocampus of PD patients is reduced or their sensitivity is reduced, patients are more prone to anxiety (64). Benzodiazepines can regulate GABA levels, which are related to the pathogenesis of $\mathrm{PD}$, but benzodiazepines have adverse effects (including drug abuse, withdrawal symptoms, and memory defects) (65). $\mathrm{PD}$ patients show decreased $\mathrm{GABA}_{\mathrm{A}}$ receptor binding, the main cause of which is to increase GABA brain levels by inhibiting the GABA-decomposing enzyme GABA transaminase (66). In addition to the above Class SSRIs and BZDs drugs, Class TCAs drugs are considered the second selected treatment such as clomiparamine is similar to SSRIs, and reduces high responsiveness to $\mathrm{CO} 2$, but have less tolerance and safety and antagonistic side effects against muscarinic, $\alpha 1$-adrenergic and histaminergic receptors (67).

\section{cAMP signaling patbway}

cAMP is a second messenger involved in cell growth, neurotransmitter release, gene transcription, and other cellular processes. PKA is an inactive tetrameric holoenzyme that depends on cAMP (68). PKA activity is affected by a variety of neurotransmitters (acetylcholine, dopamine, norepinephrine, and serotonin). These transmitters activate adenylate cyclase activity through $G$ proteins, induce ATP to become cAMP, and participate in the regulation of anxiety and fear emotions. Therefore, the cAMP-PKA signaling pathway plays an important role in fear (69). Injection of a cAMP-like drug (bucladesine) into the hippocampus of rats can alleviate the fear memory deficits caused by PKA inhibitors (H-89), and the cAMPPKA signaling pathway plays an important role in memory consolidation in the fear conditioned response model (70). Targeted activation of cAMP-PKA signaling in the lateral amygdala increases the excitability of its neurons, leading to widespread fear. This observation provides new ideas for further research on the role of amygdala cells and pathways in fear (71). Inhibition of phosphodiesterase 4 (PDE4) causes an increase in cAMP, which in turn activates PKA. PDE4 inhibitors activate the cAMP-PKA pathway, which can protect and regenerate nerves, have an antiinflammatory effect, and enhance memory and cognition. PDE4 inhibitors are used to treat anxiety, depression, and cognitive disorders (72).

In addition, key targets are related to inflammation and tumor response. KEGG pathway analysis of QZF can be effective through cancer pathway, related to colorectal cancer and prostate cancer, reflecting the characteristics of different diseases of traditional Chinese medicine. Because network pharmacology has the characteristics of drug measurement, the mechanism of some TCM treatment diseases may find that the treatment of another disease, before the drug or leading compounds for the new disease, so QZF not only through multiple mechanism treatment of $\mathrm{PD}$ can provide certain clues for the treatment of inflammation, tumor and drug use, but still need further clinical and experimental research (73).

\section{Conclusions}

In this study, we predicted the active ingredients, targets, and mechanisms of action of QZF in the treatment of PD through network pharmacology and molecular docking methods. After analyzing the results, we believe that the 5-HT, GABA, and cAMP signaling pathways are important routes by which QZF treats $\mathrm{PD}$, meaning that QZF might have the characteristics of multicomponent, multitarget, and multipathway synergistic effects in the treatment of PD, providing certain clues for the treatment of inflammatory tumors and drug use.

\section{Acknowledgments}

Funding: Grants supported this study from the National Natural Science Foundation of China (No. 81774249), and the Shandong Science and Technology Development Plan of Traditional Chinese Medicine (No. 2019-0224).

\section{Footnote}

Conflicts of Interest: All authors have completed the ICMJE uniform disclosure form (available at https://dx.doi. org/10.21037/atm-21-4090). All authors report funding support from the National Natural Science Foundation of China (No. 81774249), and Shandong science and 
technology development plan of traditional Chinese medicine (No. 2019-0224). The authors have no other conflicts of interest to declare.

Ethical Statement: The authors are accountable for all aspects of the work in ensuring that questions related to the accuracy or integrity of any part of the work are appropriately investigated and resolved.

Open Access Statement: This is an Open Access article distributed in accordance with the Creative Commons Attribution-NonCommercial-NoDerivs 4.0 International License (CC BY-NC-ND 4.0), which permits the noncommercial replication and distribution of the article with the strict proviso that no changes or edits are made and the original work is properly cited (including links to both the formal publication through the relevant DOI and the license). See: https://creativecommons.org/licenses/by-nc-nd/4.0/.

\section{References}

1. Domhardt M, Letsch J, Kybelka J, et al. Are Internetand mobile-based interventions effective in adults with diagnosed panic disorder and/or agoraphobia? A systematic review and meta-analysis. J Affect Disord 2020;276:169-82.

2. Schumacher J, Kristensen AS, Wendland JR, et al. The genetics of panic disorder. J Med Genet 2011;48:361-8.

3. Roy-Byrne PP, Craske MG, Stein MB. Panic disorder. Lancet 2006;368:1023-32.

4. Samimi Ardestani SM, Khosravani V, Sharifi Bastan F, et al. The Persian Version of the COVID-19 Phobia Scale (Persian-C19P-S) and the differences in COVID-19related phobic reactions in patients with anxiety disorders. Int J Ment Health Addict 2021. [Epub ahead of print]. doi: 10.1007/s11469-021-00523-0.

5. Yang Y, Islam MS, Wang J, et al. Traditional Chinese medicine in the treatment of patients infected with 2019new coronavirus (SARS-CoV-2): a review and perspective. Int J Biol Sci 2020;16:1708-17.

6. Xiong TW, Wu Q, Liu J, et al. Research progress of effect of anxiolytic traditional Chinese medicines and formulas on neurotransmitters. Zhongguo Zhong Yao Za Zhi 2020;45:14-9.

7. Luo TT, Lu Y, Yan SK, et al. Network pharmacology in research of Chinese medicine formula: methodology, application and prospective. Chin J Integr Med 2020;26:72-80.

8. Ru J, Li P, Wang J, et al. TCMSP: a database of systems pharmacology for drug discovery from herbal medicines. J Cheminform 2014;6:13.

9. Xu X, Zhang $\mathrm{W}$, Huang $\mathrm{C}$, et al. A novel chemometric method for the prediction of human oral bioavailability. Int J Mol Sci 2012;13:6964-82.

10. Liu Z, Guo F, Wang Y, et al. BATMAN-TCM: a Bioinformatics Analysis Tool for Molecular mechANism of Traditional Chinese Medicine. Sci Rep 2016;6:21146.

11. UniProt Consortium. UniProt: the universal protein knowledgebase in 2021. Nucleic Acids Res 2021;49:D480-9.

12. Xu HY, Zhang YQ, Liu ZM, et al. ETCM: an encyclopaedia of traditional Chinese medicine. Nucleic Acids Res 2019;47:D976-82.

13. Kim S, Chen J, Cheng T, et al. PubChem in 2021: new data content and improved web interfaces. Nucleic Acids Res 2021;49:D1388-95.

14. Gfeller D, Michielin O, Zoete V. Shaping the interaction landscape of bioactive molecules. Bioinformatics 2013;29:3073-9.

15. Safran M, Chalifa-Caspi V, Shmueli O, et al. Human Gene-Centric Databases at the Weizmann Institute of Science: GeneCards, UDB, CroW 21 and HORDE. Nucleic Acids Res 2003;31:142-6.

16. Amberger JS, Bocchini CA, Scott AF, et al. OMIM.org: leveraging knowledge across phenotype-gene relationships. Nucleic Acids Res 2019;47:D1038-43.

17. Davis AP, Grondin CJ, Johnson RJ, et al. Comparative Toxicogenomics Database (CTD): update 2021. Nucleic Acids Res 2021;49:D1138-43.

18. Wishart DS, Feunang YD, Guo AC, et al. DrugBank 5.0: a major update to the DrugBank database for 2018. Nucleic Acids Res 2018;46:D1074-82.

19. Szklarczyk D, Gable AL, Lyon D, et al. STRING v11: protein-protein association networks with increased coverage, supporting functional discovery in genomewide experimental datasets. Nucleic Acids Res 2019;47:D607-13.

20. Shannon P, Markiel A, Ozier O, et al. Cytoscape: a software environment for integrated models of biomolecular interaction networks. Genome Res 2003;13:2498-504.

21. O'Boyle NM, Banck M, James CA, et al. Open Babel: An open chemical toolbox. J Cheminform 2011;3:33.

22. Berman HM, Westbrook J, Feng Z, et al. The Protein Data Bank. Nucleic Acids Res 2000;28:235-42.

23. Seeliger D, de Groot BL. Ligand docking and binding site analysis with PyMOL and Autodock/Vina. J Comput 
Aided Mol Des 2010;24:417-22.

24. Morris GM, Huey R, Lindstrom W, et al. AutoDock4 and AutoDockTools4: Automated docking with selective receptor flexibility. J Comput Chem 2009;30:2785-91.

25. Trott O, Olson AJ. AutoDock Vina: improving the speed and accuracy of docking with a new scoring function, efficient optimization, and multithreading. J Comput Chem 2010;31:455-61.

26. Huang da W, Sherman BT, Lempicki RA. Systematic and integrative analysis of large gene lists using DAVID bioinformatics resources. Nat Protoc 2009;4:44-57.

27. Arnold PD, Zai G, Richter MA. Genetics of anxiety disorders. Curr Psychiatry Rep 2004;6:243-54.

28. Qiu ZK, Liu CH, Gao ZW, et al. The inulin-type oligosaccharides extract from morinda officinalis, a traditional Chinese herb, ameliorated behavioral deficits in an animal model of post-traumatic stress disorder. Metab Brain Dis 2016;31:1143-9.

29. Zheng SL, Wang YH, Wu ZH, et al. Effect of Chinese yam on thymus spleen histological structure of aging-mice. Chinese Journal of Gerontology 2007;27:1881-2.

30. Jung JW, Yoon BH, Oh HR, et al. Anxiolytic-like effects of Gastrodia elata and its phenolic constituents in mice. Biol Pharm Bull 2006;29:261-5.

31. Hu Y, Liao HB, Dai-Hong G, et al. Antidepressant-like effects of 3,6'-disinapoyl sucrose on hippocampal neuronal plasticity and neurotrophic signal pathway in chronically mild stressed rats. Neurochem Int 2010;56:461-5.

32. Jin Z, Yan ZJ, Li YQ, et al. Effect of "Qiangzhizufang" on the rat model of Tourette syndrome combined with fear. Chinese Journal of Comparative Medicine 2016;26:71-6, 82 .

33. Dang DK, Duong CX, Nam Y, et al. Inhibition of protein kinase $(\mathrm{PK}) \mathrm{C} \delta$ attenuates methamphetamine-induced dopaminergic toxicity via upregulation of phosphorylation of tyrosine hydroxylase at Ser40 by modulation of protein phosphatase 2A and PKA. Clin Exp Pharmacol Physiol 2015;42:192-201.

34. Briggs GD, Nagy GM, Dickson PW. Mechanism of action of salsolinol on tyrosine hydroxylase. Neurochem Int 2013;63:726-31.

35. Zhen JL, Chang YN, Qu ZZ, et al. Luteolin rescues pentylenetetrazole-induced cognitive impairment in epileptic rats by reducing oxidative stress and activating PKA/CREB/ BDNF signaling. Epilepsy Behav 2016;57:177-84.

36. Lee B, Yeom M, Shim I, et al. Protective effects of quercetin on anxiety-like symptoms and neuroinflammation induced by lipopolysaccharide in rats. Evid Based Complement Alternat Med 2020;2020:4892415.

37. Sharma L, Sharma A, Dash AK, et al. A standardized polyherbal preparation POL-6 diminishes alcohol withdrawal anxiety by regulating Gabra1, Gabra2, Gabra3, Gabra4, Gabra5 gene expression of GABAA receptor signaling pathway in rats. BMC Complement Med Ther 2021;21:13.

38. Liu WZ, Huang BW, You WJ, et al. Harmine enhances GABAergic transmission onto basoamygdala projection neurons in mice. Brain Res Bull 2018;137:294-300.

39. Hers I, Vincent EE, Tavaré JM. Akt signalling in health and disease. Cell Signal 2011;23:1515-27.

40. Wong H, Levenga J, LaPlante L, et al. Isoform-specific roles for AKT in affective behavior, spatial memory, and extinction related to psychiatric disorders. Elife 2020;9:56630.

41. Riedel M, Berthelsen MF, Cai H, et al. In vivo CRISPR inactivation of Fos promotes prostate cancer progression by altering the associated AP-1 subunit Jun. Oncogene 2021;40:2437-47.

42. Hein S, Mahner S, Kanowski C, et al. Expression of Jun and Fos proteins in ovarian tumors of different malignant potential and in ovarian cancer cell lines. Oncol Rep 2009;22:177-83.

43. Chen TK, Smith LM, Gebhardt DK, et al. Activation and inhibition of the AP-1 complex in human breast cancer cells. Mol Carcinog 1996;15:215-26.

44. Matthiesen M, Spiacci A Jr, Zangrossi H Jr. Effects of chemical stimulation of the lateral wings of the dorsal raphe nucleus on panic-like defensive behaviors and Fos protein expression in rats. Behav Brain Res 2017;326:103-11.

45. Pockros LA, Pentkowski NS, Conway SM, et al. 5-HT(2A) receptor blockade and 5-HT(2C) receptor activation interact to reduce cocaine hyperlocomotion and Fos protein expression in the caudate-putamen. Synapse 2012;66:989-1001.

46. Takahashi S, Fukushima H, Yu Z, et al. Tumor necrosis factor $\alpha$ negatively regulates the retrieval and reconsolidation of hippocampus-dependent memory. Brain Behav Immun 2021;94:79-88.

47. Hall J, Thomas KL, Everitt BJ. Fear memory retrieval induces CREB phosphorylation and Fos expression within the amygdala. Eur J Neurosci 2001;13:1453-8.

48. Song X, Liu B, Cui L, et al. Silibinin ameliorates anxiety/ depression-like behaviors in amyloid $\beta$-treated rats by upregulating BDNF/TrkB pathway and attenuating 
autophagy in hippocampus. Physiol Behav 2017;179:487-93.

49. Belem da Silva CT, Costa MA, Bortoluzzi A, et al. Cytokine Levels in Panic Disorder: Evidence for a DoseResponse Relationship. Psychosom Med 2017;79:126-32.

50. Taniguchi K, Karin M. IL-6 and related cytokines as the critical lynchpins between inflammation and cancer. Semin Immunol 2014;26:54-74.

51. Klaus F, Paterna JC, Marzorati E, et al. Differential effects of peripheral and brain tumor necrosis factor on inflammation, sickness, emotional behavior and memory in mice. Brain Behav Immun 2016;58:310-26.

52. Locksley RM, Killeen N, Lenardo MJ. The TNF and TNF receptor superfamilies: integrating mammalian biology. Cell 2001;104:487-501.

53. Gobejishvili L, Barve S, Joshi-Barve S, et al. Chronic ethanol-mediated decrease in cAMP primes macrophages to enhanced LPS-inducible NF-kappaB activity and TNF expression: relevance to alcoholic liver disease. Am J Physiol Gastrointest Liver Physiol 2006;291:G681-8.

54. Bocchio M, McHugh SB, Bannerman DM, et al. Serotonin, Amygdala and Fear: Assembling the Puzzle. Front Neural Circuits 2016;10:24.

55. Waider J, Popp S, Lange MD, et al. Genetically driven brain serotonin deficiency facilitates panic-like escape behavior in mice. Transl Psychiatry 2017;7:e1246.

56. Spannuth BM, Hale MW, Evans AK, et al. Investigation of a central nucleus of the amygdala/dorsal raphe nucleus serotonergic circuit implicated in fear-potentiated startle. Neuroscience 2011;179:104-19.

57. Jennings KA, Lesch KP, Sharp T, et al. Non-linear relationship between 5 -HT transporter gene expression and frequency sensitivity of 5-HT signals. J Neurochem 2010;115:965-73.

58. Westenberg HG, Liebowitz MR. Overview of panic and social anxiety disorders. J Clin Psychiatry 2004;65 Suppl 14:22-6.

59. Ziffra M. Panic disorder: A review of treatment options. Ann Clin Psychiatry 2021;33:124-33.

60. Kita H, Kitai ST. Glutamate decarboxylase immunoreactive neurons in rat neostriatum: their morphological types and populations. Brain Res 1988;447:346-52.

61. da Silva JA, Almada RC, de Figueiredo RM, et al. Blockade of synaptic activity in the neostriatum and activation of striatal efferent pathways produce opposite effects on panic attack-like defensive behaviours evoked by GABAergic disinhibition in the deep layers of the superior colliculus. Physiol Behav 2018;196:104-11.

62. Santos M, D'Amico D, Spadoni O, et al. Hippocampal hyperexcitability underlies enhanced fear memories in TgNTRK3, a panic disorder mouse model. J Neurosci 2013;33:15259-71.

63. Medel-Matus JS, Shin D, Sankar R, et al. Kindling epileptogenesis and panic-like behavior: Their bidirectional connection and contribution to epilepsyassociated depression. Epilepsy Behav 2017;77:33-8.

64. Roy-Byrne PP. The GABA-benzodiazepine receptor complex: structure, function, and role in anxiety. J Clin Psychiatry 2005;66 Suppl 2:14-20.

65. Masdrakis VG, Baldwin DS. Anticonvulsant and antipsychotic medications in the pharmacotherapy of panic disorder: a structured review. Ther Adv Psychopharmacol 2021;11:20451253211002320.

66. Malizia AL, Cunningham VJ, Bell CJ, et al. Decreased brain GABA(A)-benzodiazepine receptor binding in panic disorder: preliminary results from a quantitative PET study. Arch Gen Psychiatry 1998;55:715-20.

67. Perna G, Guerriero G, Caldirola D. Emerging drugs for panic disorder. Expert Opin Emerg Drugs 2011;16:631-45.

68. Skalhegg BS, Tasken K. Specificity in the cAMP/PKA signaling pathway. Differential expression,regulation, and subcellular localization of subunits of PKA. Front Biosci 2000;5:D678-93.

69. Arnsten AF, Ramos BP, Birnbaum SG, et al. Protein kinase $A$ as a therapeutic target for memory disorders: rationale and challenges. Trends Mol Med 2005;11:121-8.

70. Nassireslami E, Nikbin P, Payandemehr B, et al. A cAMP analog reverses contextual and tone memory deficits induced by a PKA inhibitor in Pavlovian fear conditioning. Pharmacol Biochem Behav 2013;105:177-82.

71. Ghosh S, Chattarji S. Neuronal encoding of the switch from specific to generalized fear. Nat Neurosci 2015;18:112-20.

72. Richter W, Menniti FS, Zhang HT, et al. PDE4 as a target for cognition enhancement. Expert Opin Ther Targets 2013;17:1011-27.

73. Ren Y, Deng YJ, Ma HB, et al. Research progress and challenges of network pharmacology in field of traditional Chinese medicine. Chinese Traditional and Herbal Drugs 2020;51:4789-97.

Cite this article as: Zhao R, Liu P, Song A, Liu J, Chu Q, Liu Y, Jiang Y, Dong C, Shi H, Yan Z. Network pharmacology study on the mechanism of Qiangzhifang in the treatment of panic disorder. Ann Transl Med 2021;9(16):1350. doi: 10.21037/atm21-4090 